\title{
CP-NR Distributed Range Free Localization Algorithm in WSN
}

\author{
Deepak Prashar ${ }^{1}$, Kiran Jyoti ${ }^{2}$, Dilip Kumar ${ }^{3}$ \\ ${ }^{1} \mathrm{CSE}$, IKGPTU, Punjab, India \\ ${ }^{2}$ Department of IT, GNDEC, Punjab, India \\ ${ }^{3}$ Department of Electronics, SLIET, Punjab, India
}

\begin{tabular}{|c|c|}
\hline Article Info & ABSTRACT \\
\hline Article history: & \multirow{10}{*}{$\begin{array}{l}\text { Advancements in wireless communication technology have empowered the } \\
\text { researchers to develop large scale wireless networks with huge number of } \\
\text { sensor nodes. In these networks localization is very active field of research. } \\
\text { Localization is a way to determine the physical position of sensor nodes } \\
\text { which is useful in many aspects such as to find the origin of events, routing } \\
\text { and network coverage. Locating nodes with GPS systems is expensive, } \\
\text { power consuming and not applicable to indoor environments. Localization in } \\
\text { three dimensional space and accuracy of the estimated location are two } \\
\text { factors of major concern. In this paper, a new three dimensional Distributed } \\
\text { range-free algorithm which is known as CP-NR is proposed. This algorithm } \\
\text { has high localization accuracy and resolved the problem of existing NR } \\
\text { algorithm. CP-NR (Coplanar and Projected Node Reproduction) algorithm } \\
\text { makes use of co-planarity and projection of point on plane concepts to reduce } \\
\text { the localization error. Results have shown that CP-NR algorithm is superior } \\
\text { to NR algorithm and comparison is done for the localization accuracy with } \\
\text { respect to variations in range, anchor density and node density. }\end{array}$} \\
\hline Received Feb 14, 2018 & \\
\hline Revised Apr 20, 2018 & \\
\hline Accepted May 27, 2018 & \\
\hline Keyword: & \\
\hline Centroid & \\
\hline DV-Hop & \\
\hline GPS & \\
\hline NR & \\
\hline WSN & \\
\hline
\end{tabular}

Copyright (c) 2018 Institute of Advanced Engineering and Science. All rights reserved.

\section{Corresponding Author:}

Deepak Prashar, CSE, IKGPTU, Punjab, India.

Email: deepakprash@gmail.com

\section{INTRODUCTION}

There are several issues in WSNs [1], [2] which are required to be considered for the construction of robust and efficient network. In Wireless Sensor Networks, sensor nodes determine their location by localization. For this purpose localization algorithms are used in WSN [3], [4], [5]. Localization is a process of estimating the position and co-ordinates of wireless sensor nodes. Localization information is important due to several factors such as the recognition of gathered data, node labeling, management and concern of localized nodes in a decided region, impact of node's density and coverage, energy draft formation, topographical routing, entity tracking, and other geographic algorithms. All these factors make localization as one of the key mechanics for the evolution and exploitation of WSN.

The nodes which are conscious of their positions either from GPS or manualy configured are known as Anchor nodes. The nodes which don't know their positions are called dumb or unknown nodes that take the help of anchor nodes to obtain their positions. Localization can be done either manualy or by using GPS. GPS is best measurement approach that obtains location directly without any further computation. But addition of GPS to all nodes in the wireless sensor network is impractical because of its high cost, high power usage and environment suppression, which makes it infeasible for indoor applications. Self-localization is a substitute of GPS. Hop count and neighborhood measurements are the techniques that make use of radio connectivity information. The area of evaluations can use either range or connectivity information based on design of area constraints.

Several techniques have been suggested for working out Localization problem [6], [7], [8] but most of them consider only for 2-dimensional network. Hence, location estimation issue in three dimensions is a 
demanding concern area in the research community. Analysis of localization approaches concentrating on the 3-D space is a significant task to support real applications of WSNs, because the difference between localization in 2-D and 3-D space is expressive. Three anchor nodes are required to determine the location of nodes in 2D, whereas in 3D, four anchor nodes are needed. 2-D spaces cannot be exactly changed to 3-D just by addition of one more parameter. There are several issues that can be smoothly resolved in the sensor network as 2-D but are very complex in 3-D [9], [10]. Moreover, the triangulation approach that is engaged in 2-D space, is not applicable 3-D.In 3D, quadrilateration approach can be used to localize a WSN that uses distance measurements of four non-coplanar sensors to find location of an unknown node in 3D. Therefore we need a cost effective and resource efficient localization algorithm for 3D WSNs. As the sensor networks are application peculiar it is truly difficult to conclude the algorithms for localization that fits best to all the different schemes.

\section{PROBLEM IDENTIFICATION}

There is a need of 3D localization [11], [12] to provide accuracy and reducing positioning error [13] in harsh as well as flat terrain spaces. The three dimensional localization is much more complicated and has more the computational complexity as a result of which it is not appropriate to extend the 2D localization algorithm to a 3D algorithm directly. The research on 3-D localization is more realistic and localization algorithms in the three-dimensional space are necessary. One of the distributed range free algorithms in 3-D based on Node Reproduction [14] is taken as the point of further research with respect to this paper. It has smaller computation overhead and provides better localization than DV-Hop and Centroid Algorithm.

In NR Algorithm [10] the localization space is supposed to be in the space of a cube which is having the length of edge to be $100 \mathrm{~m}$. The total volume of the 3D localization space is $100 \times 100 \times 100$ cubic meter. There are 216 anchor nodes considered in this localization technique. Form each one of a cube, every eight anchor nodes having edge length $20 \mathrm{~m}$ in the space. All the unknown nodes are deployed randomly. Area of communication of each unknown node is another important parameter. When the packets sent by anchor nodes enter into the communication range of any unknown node, packets are detected immediately and unknown node records the corresponding anchor node's information including anchor node ID and coordinates. Then unknown node can obtain reproduced node using location information of three different anchor nodes and at the end the unknown node's coordinates are obtained by the use of three anchors and one reproduced node.

NR algorithm [14] has following steps:

a. The algorithm begins by broadcasting data packets from anchors to the whole network periodically. For this time duration $(\mathrm{T})$ can be set to manual. Anchor node ID, and its co-ordinates are the main constituents of the data that is broadcasted.

b. Unknown nodes just listen to these packages that are sent in the time duration $(T)$. Unknown nodes need to record number of packets they have received from different anchors.

c. Check whether the time duration $(\mathrm{T})$ is arrived or not. If arrived then information to be recorded, otherwise it will go on waiting.

d. After recording anchor nodes information is, unknown node computes reproduced node with the help of three anchor nodes.

e. Based on recorded three anchor nodes information unknown node predict a fourth anchor node's position on the same plane with the help of three anchor nodes by formulating a square with edge length $20 \mathrm{~m}$.

f. Using these four nodes, the square center point is computed. Reproduced node differs from the center node by means of one different cordinate direction and the other two are in the same.

g. Reproduced node is estimated by addition of half communication range to any one of three cordinate directions.

h. After generation of reproduced node, unknown nodes end the localization process. Three anchors and reproduced node forms a tetrahedron. Then by using similar way of centroid algorithm, the center of these four nodes estimates position of the unknown node.

NR algorithm doesn’t require sensors having any special range-determining hardware. It depends on node-anchor communication to find the location of the unknown nodes that reduces the computational overhead of the whole network. Moreover the communication overhead is reduced which prolongs the whole network’s lifetime.

In this algorithm there are some problems in which future work is considered to be done:

a. Position of the Reproduced Node: After determination of reproduced node, it is not sure about its plane in which it is generated. It may lie on the same plane with unknown node or in the opposite plane. If it is in opposite plane with respect to the unknown node, localization error is produced. During localization process, the reproduced node's position is calculated randomly which is not a best solution and cause 
number of uncertainties in the network. How to resolve this issue is a future research area in respect to NR [14] which is worked upon in the proposed algorithm below.

b. Communication Range: In NR algorithm, the small communication range leads to incompletion of location estimations. Because of this low value of communication range, the covered sensor area is small which pose as an obstacle in the generation of reproduced node. Whereas large communication range provides highly accurate estimations, but in this case the packets from different anchors get conflicted and this cannot produce accurate estimation. This issue is also taken in to account in the propsed approach.

\section{PROPOSED WORK}

CP-NR technique has tried to reduce the problem of localization error due to wrong position of reproduced node as mentioned in the above section. In this method, the localization space is a cube of volume $100 \times 100 \times 100$ cubic meter. There are total 400 nodes out of which 216 anchor nodes and rest are unknown nodes. A cube having edge length $20 \mathrm{~m}$ is formed using eight anchors in which randomly deployed unknown nodes exists. The communication range in this algorithm is $30 \mathrm{~m}$ also. This algorithm generates reproduced node same as NR algorithm. Anchor nodes broadcast messages in random threshold time duration (T). Unknown nodes listen messages and check whether time duration $(\mathrm{T})$ has arrived. If time duration $(\mathrm{T})$ is arrived, unknown nodes record information of three anchor nodes which have maximum beacon transmission. Using this information unknown node predict a fourth one anchor node on to make a square of side $20 \mathrm{~m}$ in the same plane. Using these four nodes, the center point of the square is computed. Reproduced node is estimated by addition of half communication range to any one of three ordinate directions of center node. But after generation of reproduced node, check on the plane in which reproduced node lies is done.

The co-planarity concept [15] of four nodes determined the position of the reproduced node.

For co-planarity, suppose three points A, B, C forms a plane and determines whether the fourth point $\mathrm{D}$ lies on the same plane. Cross product of two vectors (Vector $\mathrm{AB} \times$ Vector $\mathrm{AC}$ ) is normal to plane. Prove that the last vector $\mathrm{AD}$ is normal to this cross product. Hence the triple product should equal to zero.

$$
\overrightarrow{A D} \cdot(\underset{A B}{\rightarrow} \times \underset{A C}{\rightarrow})=0
$$

If reproduced node does not lie within the same plane that of recorded three anchors nodes then need to use projection of point technique to put this node in the appropriate plane to increase the localization accuracy. And at the end three anchors and reproduced node forms a tetrahedron. Then by using similar way of centroid algorithm, the center of these four nodes estimates position of the unknown node.

Projection of a point on a plane: Let $\pi$ be any plane and $\mathrm{P}$ be a given point, not on the plane $\pi$, then $\mathrm{P}$ ' the foot of perpendicular on the plane, is called orthogonal projection of $\mathrm{P}$ on the plane $\pi$.

\section{RESULTS AND ANALYSIS}

In the proposed CP-NR algorithm localization error due to incorrect position of reproduced node is reduced using the concepts of co-planarity and projection of a point on the plane which determine the plane of the reproduced node with respect to recorded anchor nodes and positions it in the appropriate plane to generate more accurate location estimation results. In these algorithms the basic node for location estimation is reproduced node. How to generate it in the appropriate plane is the main concern. Generation of the reproduced node in the opposite plane produced high localization error. Here to reduce that localization error first check the existence of generated reproduced node with respect to the anchor nodes that is whether it is in the same plane of the anchor nodes or not, with the help of co-planarity concept. If it is found in some another plane with respect to anchor nodes from which it is generated, then projection of a point on the plane concept is used to place it in the same plane of anchor nodes. All the simulation work is done in the MATLAB software, a programming language that provides interactive visualization, mathematical computations and programming environment.

In the first sub section 1, steps related to deployment with respect to existing NR and proposed CP-NR algorithms is executed. These steps include design of network space, density and deployment of anchor and unknown nodes, selection of anchor nodes and generation of the reproduced node for the localization process. In the second sub section 2, experimental results of localization errors in done for the both algorithms. This section shows that how the proposed algorithm is better than the existing one in the terms of localization accuracy. In the third sub section 3, the comparison among the proposed CP-NR and the existing NR algorithm on the basis of localization error is done. Then the effect of several parameters such as anchor density, node density and range parameter on the results of these algorithms is executed. 


\subsection{Sub Section 1}

First of all nodes are deployed in 3D environment having the cube of edge length $100 \mathrm{~m}$ and having total nodes 400, out of which 216 is the anchor's density. The total localization space volume is $100 * 100 * 100$ cubic meter in which the unknown nodes are randomly deployed. Figure 1 represents the cubical 3D localization space in which red colored 216 anchor nodes and (400-216) black colored unknown nodes are deployed.

The anchor nodes broadcast beacon information periodically which is listened and recorded by the unknown nodes lie in their range. This information is recorded in the descending order which forms the basis of anchor node selection. Figure 2 represents the selection of anchor nodes on the basis of their maximum recorded information parameter at unknown nodes in cubical 3D localization space which having 216 red colored anchor and (400-216) black colored unknown nodes. Now selection of three anchor nodes from which unknown nodes received maximum beacon information is done.

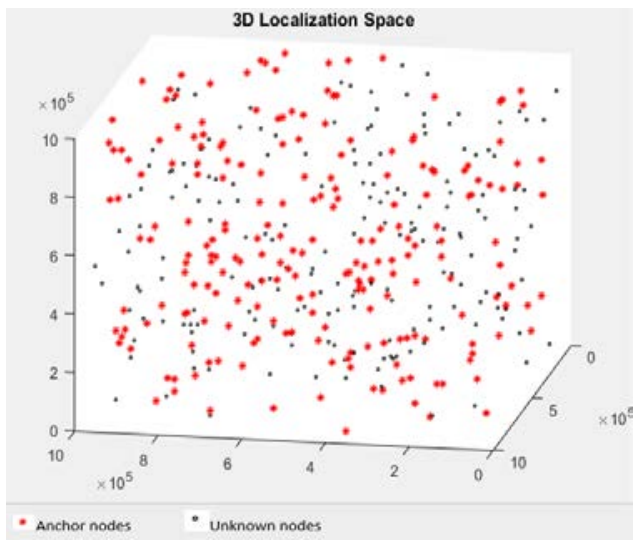

Figure 1. Localization space in NR and CP-NR algorithms

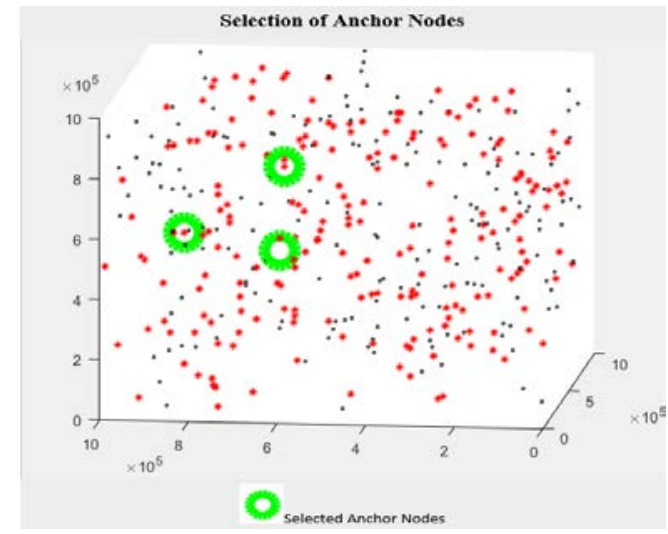

Figure 2. Selection of three anchor nodes

The generation of the reproduced node is the crucial aspect of both NR and CP-NR algorithms. It helps in the location estimation of unknown nodes. The three selected anchor nodes and this reproduced node form a tetrahedron and use centroid concept to end the localization process of these algorithms. The generation of the reproduced node makes effect on the communication range to cover the whole network as well as the density of the anchor nodes. Figure 3 represents the generation of reproduced node using four anchor nodes: three selected anchor nodes and one determined anchor node. This fourth anchor node is selected by unknown node having same plane that of three selected anchors. These anchor nodes form a square plane from which reproduced node is generated. In the Figure 4, four anchor nodes are of green color and reproduced node is of blue color.

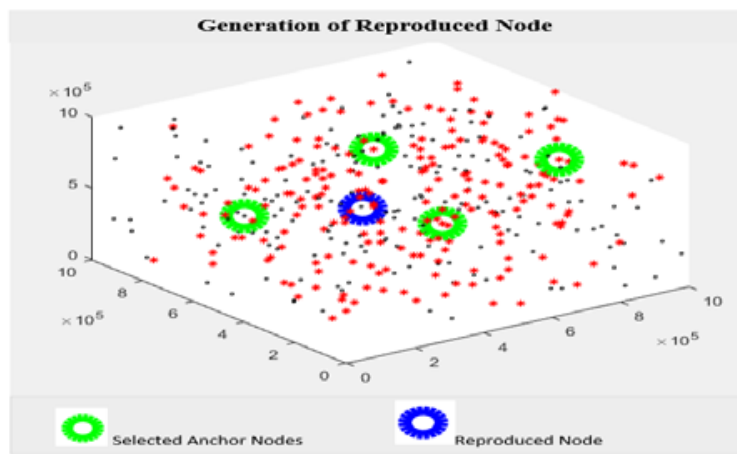

Figure 3. Generation of Reproduced Node 


\subsection{Sub Section 2}

The results of the localization errors in NR algorithm in both the scenarios when the reproduced node lie within the same plane of anchor nodes and when it lies in the opposite plane are presented here. When the reproduced lies within the same plane of selected anchors then localization error is less. But opposite to this when it is not in the same plane it produces more inaccurate position estimations of the unknown nodes. Figure 4 represents the localization error when the reproduced node lies in the accurate plane and Figure 5 represents the localization error when reproduced node lies in the opposite plane.

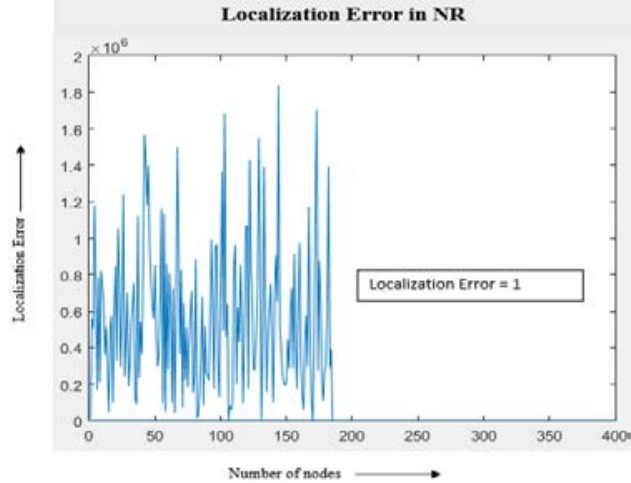

Figure 4. Error with reproduced node in appropriate plane

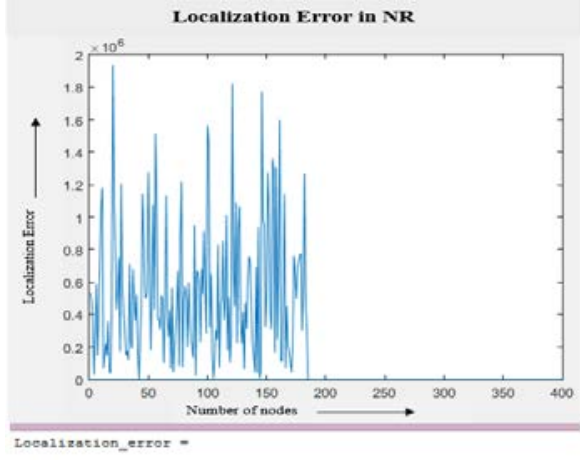

Figure 5. Error when reproduced node in the opposite plane

The problem of high localization error in the existing NR algorithm when reproduced node in some other plane has been addressed in the proposed CP-NR algorithm. After the generation of it, its position with respect to the selected anchors is examined using co-planarity concept of four anchor nodes. If it is in some other plane then it is to be brought on to the accurate plane using projection of a point on the plane concept. This concept projects the co-ordinates of reproduced node in the accurate plane. In this way CP-NR reduces the error in localization and provides accurate position estimations of the unknown nodes. Figures 5 and 6 represent the localization error in the CP-NR algorithm.

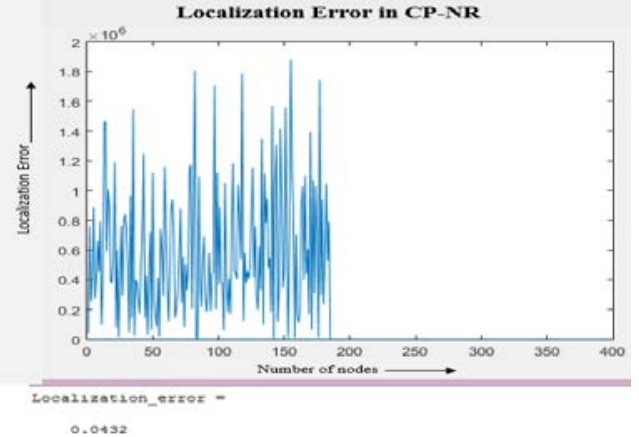

Figure 6. Localization error in CP-NR algorithm

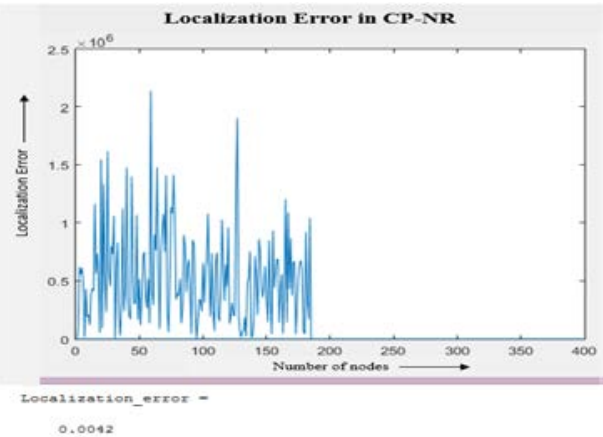

Figure 7. Localization error in CP-NR algorithm

\subsection{Sub Section 3}

The CP-NR algorithm eliminates the problem of existing NR algorithm that is high localization error due to the inaccurate plane of the reproduced node. In the proposed algorithm the position of reproduced node is always correct which leads to the high localization accuracy. The appropriate plane of reproduced node is obtained whenever it lies in another plane using projection of its co-ordinates on the plane. Table1 below contains the five values of localization error estimations randomly in the both CP-NR and NR algorithms. 
Table 1. Localization Error Estimations of NR and CP-NR Algorithms

\begin{tabular}{ccc}
\hline & \multicolumn{2}{c}{ Localization error } \\
Number of Observations & Existing NR & Proposed CP-NR \\
\hline 1 & 1 & 0.0874 \\
2 & 14 & 0.2552 \\
3 & 34 & 0.5503 \\
4 & 57 & 0.8667 \\
5 & 63 & 1.3433 \\
\hline
\end{tabular}

Figures 8 and 9 represent the comparison among both algorithms in terms of localization accuracy. This describes high localization accuracy of CP-NR algorithm as compared to existing NR. In these figures five random numbers of observations are taken to take the localization error estimations of both proposed CP-NR and existing NR algorithm. After that these estimations are mapped into percentages of localization errors with respect to number of observations. Both the graphs are giving representation of localization accuracy. CP-NR algorithm has less localization error estimations which lead to high accuracy as compared to NR algorithm as shown in the figures below:

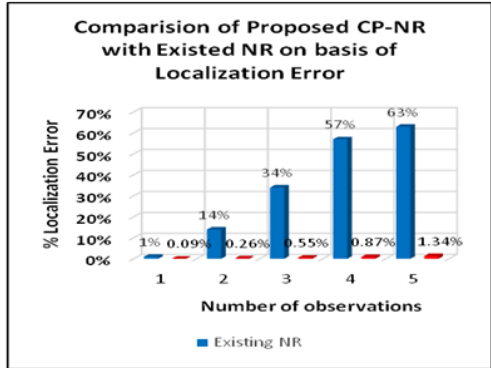

Figure 8. Comparison of CP-NR with existing NR

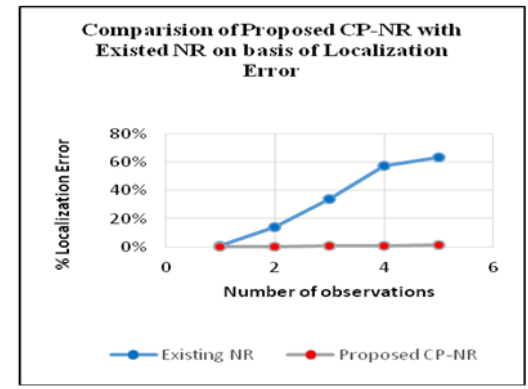

Figure 9. Comparison of CP-NR with existing NR

In this section below comparision based on the impact of several parameters such as anchor density, total node density and range on the localization accuracy on both CP-NR and existed NR algorithm is done.

Here three localization error estimations are taken to compute the average localization error at each range of anchor nodes. Figures 10 and 11 described that how the localization accuracy varies as count of anchor nodes are changed in the network. From the results of these computations it has been observed that NR algorithm gives accurate estimations with medium (250-350) of anchor nodes. But CP-NR produces accurate estimations at low and high value of anchor node density. Figure 12 represents the localization accuracy comparisons among both the algorithms on the basis of anchor node density.

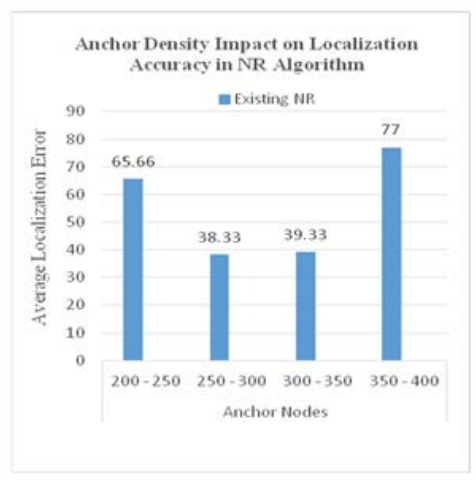

Figure 10. Anchor Density in NR

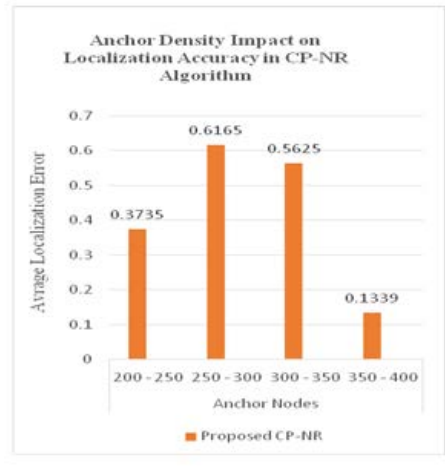

Figure 11. Anchor Density in CP-NR

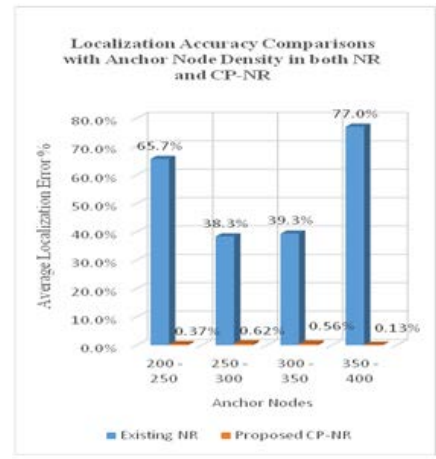

Figure 12. Comparision of NR and CP-NR 
To study the total node density impact on the localization error in both algorithms, fixed number of anchors i.e 216 are taken. Then varied the total number of nodes which gives the number of unknown nodes (total nodes - anchor nodes) in the network. Figures 13 and 14 represents total node density impact on the localization error in the existing NR and proposed CP-NR algorithms. From these graphs it is concluded that NR algorithm performs well in high node density but the CP-NR algorithm works well in high as well as low node density scenarios. Figure 15 represents the localization accuracy comparisons among NR and CP-NR on the basis of total node density.

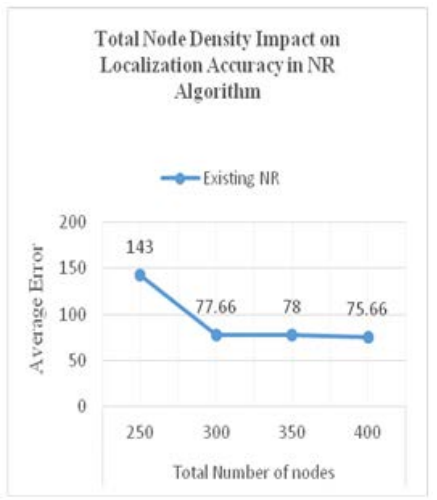

Figure 13. Accuracy in NR

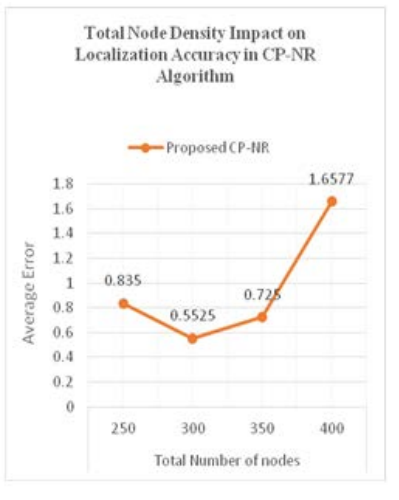

Figure 14. Accuracy in CP-NR

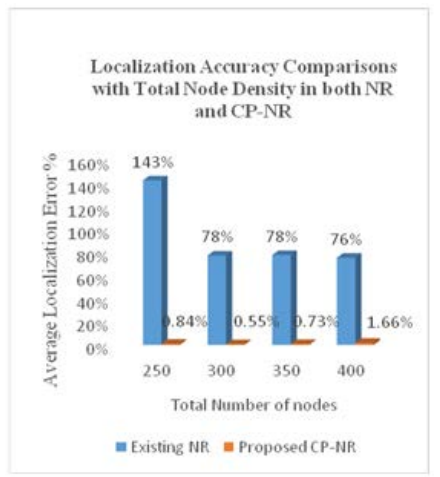

Figure 15. NR and CP-NR comparision

The generation of the reproduced node makes effect on the communication range to cover the whole network. Figures 16 and 17 described the localization error variations with different range parameters. Both of them conclude that the NR and CP-NR algorithms give best estimation results at range value $30 \mathrm{~m}$. In these algorithms the edge length is $20 \mathrm{~m}$ so with range value $30 \mathrm{~m}$ the unknown nodes can listen more beacons from the anchor nodes and large localization area is covered. Both the algorithms have one common problem of the range parameter. At lower values of range less than $10 \mathrm{~m}$ both the algorithms can't complete localization process. The reason is that the less network area coverage which destructs the generation of reproduced node and causes loss of beacons broadcasted by anchors.

When the range is higher than $30 \mathrm{~m}$ the beacons from anchors are conflicted and area provided by selected anchor nodes for localization cannot achieve the best. To make both the algorithms optimum how to solve the problem of range parameter is of future concern. Figure 18 represents the localization comparisons among both NR and CP-NR on the basis of range parameter

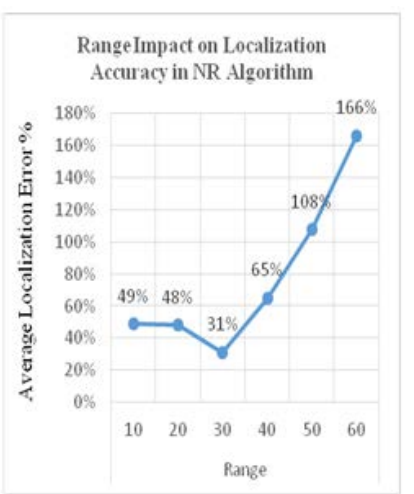

Figure 16. Range Parameter in NR

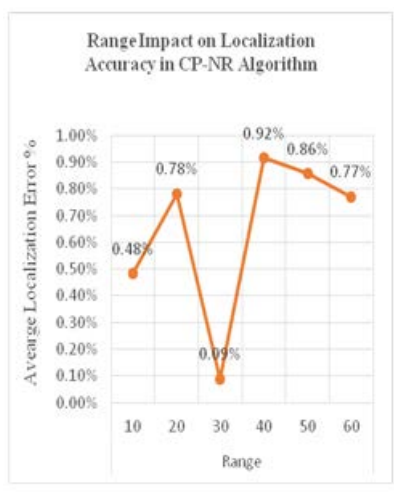

Figure 17. Range Parameter in CP-NR.

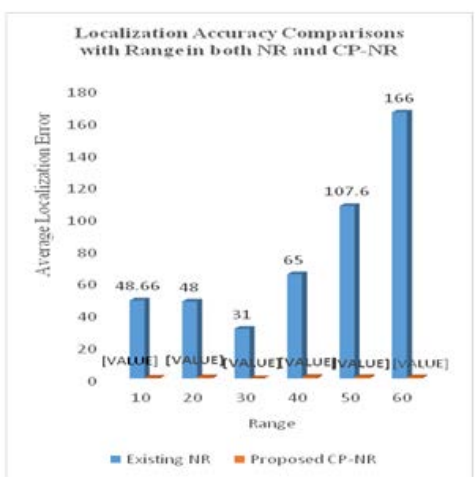

Figure 18. NR and CP-NR range comparision 


\section{CONCLUSION}

The design of a localization algorithm in wireless sensor networks is an important task. Because of geographic correlation of perceived data, location information is used to address nodes and regions, to identify the gathered data and to increase the performance of many geographic algorithms. Accuracy in estimated location is a key driver for localization process. In this paper a new distributed localization algorithm known as CP-NR is proposed. This algorithm provides high localization accuracy and resolves the problem related to the position of reproduced node. This study describes the concepts of co-planarity and projection of a point on the plane to generate reproduced node in the appropriate plane. The use of these methods significantly improves the location estimation accuracy for unknown nodes. The simulations showed that CP-NR algorithm outperforms the NR algorithm in terms of localization accuracy. Variations in localization accuracy of CP-NR and NR algorithms on the basis of different parameters such as anchor density, node density and range is also been done. As the energy consumption of nodes is critical in WSNs, then energy efficient design of CP-NR for mobile node scenarios with immense values of range are the research directions for the future.

\section{REFERENCES}

[1] F. Akyildiz, W. Su. Wireless sensor Networks: A survey, Computer Networks, 2005;38(4): 393-422.

[2] Lovepreet Singh.Techniques of Node Localization in Wireless Sensor Networks:A Review, International Journal of Innovative Research in Computer and Communication Engineering, ISO 3297: 2007 Certified Organization,2014;2(5):4143-4148.

[3] Pradnya Gajbhiye, Anjali Mahajan. A Survey of Architecture and Node deployment in WSN, IEEE Conference application of digital information and Web ,2008:426-430.

[4] Amita Sharma, Yogita Wadhwa. Routing and Computing in Wireless, International Journal of Advanced Research in Computer Science and Software Engineering,2013;3(1):336-339.

[5] B. R. Stojkoska, A. P. Avramova, and P. Chatzimisios. Application of wireless sensor networks for indoor temperature regulation, International Journal of Distributed Sensor Networks,2014;10:1-10.

[6] Rania Khadim, Mohammed Erritali, Abdelhakim Maaden. Rang-Free Localization Schemes for Wireless Sensor Networks, TELKOMNIKA Indonesian Journal of Electrical Engineering, 2015; 16(2):323-332.

[7] Chandirasekaran D, T. Jayabarathi. Wireless Sensor Networks Node Localization-A Performance Comparison of Shuffled Frog Leaping and Firefly Algorithm in LabVIEW, TELKOMNIKA Indonesian Journal of Electrical Engineering, 2015; 14(3):516-524.

[8] Yang Sun, Shoulin Yin, Jie Liu. Novel DV-hop Method Based on Krill Swarm Algorithm Used for Wireless Sensor Network Localization, TELKOMNIKA, 2016; 14(4): 1438-1445.

[9] Shayon Samanta , Prof. Punesh U.Tembhare. A Survey on 3d Localization in Wireless Sensor Networks, International Journal Of Computational Engineering Research, 2013;3(1):90-94.

[10] Q.Shi, H. Huo, T. Fang. A 3D node localization scheme for wireless sensor networks, IEICE Electronics Express,2009;6(3):167-172.

[11] Quan LIU, Ping REN, Zude ZHOU. Three-dimensional Accurate Positioning Algorithm based on Wireless Sensor Networks, Journal of Computers,2011;6(12)

[12] P.K. Singh. Node Localization in Wireless Sensor Networks, International Journal of Computer Science and Information Technologies, 2011; 2(6): 2568- 2572.

[13] [13] Sudha H Thimmaiah, Mahadevan G. A Range Based Localization Error Minimization Technique for Wireless Sensor Network, Indonesian Journal of Electrical Engineering and Computer Science, 2017; 7(2):395403.

[14] Xiaoming Wu, Yang Liu, Jianping Xing. Node Reproduction Based Range-free Localization Algorithm in Wireless Sensor Networks, Journal of Computes,2014; 9(5):1047-1052.

[15] Dinesh Khattar. Coplanarity of four points, The Pearson Guide To Mathematics For The Iit-Jee, 3/E, Dorling Kindersley (India) Pvt. Ltd. licensees of Pearson Education in South Asia, 2010:4-6. 